\title{
Effects of vertical shear in modelling horizontal oceanic dispersion
}

\author{
A. S. Lanotte ${ }^{1,2}$, R. Corrado ${ }^{3}$, L. Palatella ${ }^{1,2}$, C. Pizzigalli ${ }^{1}$, I. Schipa ${ }^{4}$, and R. Santoleri ${ }^{3}$ \\ ${ }^{1}$ CNR ISAC, GOS Team, Str. Prov. Lecce Monteroni, 73100 Lecce, Italy \\ ${ }^{2}$ INFN, Sez. di Lecce, 73100 Lecce, Italy \\ ${ }^{3}$ CNR ISAC, GOS Team, Via Fosso del Cavaliere 1, 00133 Rome, Italy \\ ${ }^{4}$ ARPA Puglia (Regional Environmental Protection Agency), Corso Trieste 27, 70126 Bari, Italy \\ Correspondence to: A. S. Lanotte (a.lanotte@isac.cnr.it)
}

Received: 29 June 2015 - Published in Ocean Sci. Discuss.: 2 September 2015

Revised: 18 January 2016 - Accepted: 19 January 2016 - Published: 3 February 2016

\begin{abstract}
The effect of vertical shear on the horizontal dispersion properties of passive tracer particles on the continental shelf of the South Mediterranean is investigated by means of observation and model data. In situ current measurements reveal that vertical gradients of horizontal velocities in the upper mixing layer decorrelate quite fast ( $\sim 1$ day), whereas an eddy-permitting ocean model, such as the Mediterranean Forecasting System, tends to overestimate such decorrelation time because of finite resolution effects. Horizontal dispersion, simulated by the Mediterranean sea Forecasting System, is mostly affected by: (1) unresolved scale motions, and mesoscale motions that are largely smoothed out at scales close to the grid spacing; (2) poorly resolved time variability in the profiles of the horizontal velocities in the upper layer. For the case study we have analysed, we show that a suitable use of deterministic kinematic parametrizations is helpful to implement realistic statistical features of tracer dispersion in two and three dimensions. The approach here suggested provides a functional tool to control the horizontal spreading of small organisms or substance concentrations, and is thus relevant for marine biology, pollutant dispersion as well as oil spill applications.
\end{abstract}

\section{Introduction}

The role of small-scale motion in geophysical flows is receiving renewed attention (Kantha and Clayson, 2000), concerning the hydrodynamical modelling, as well as in relation to the biological consequences of specific phenomena (see e.g. Durham and et al., 2013). Tracer dispersion in the ocean (Davis, 1983) has an impact on different environmen- tal, chemical, biological, and technological problems. Mean currents mostly contribute to the large-scale transport, while small-scale motions tend to spread concentration fields or, equivalently, Lagrangian trajectories of passive or active tracers. Very little is known about the way turbulence and diffusion - in addition to other physical mechanisms - model marine habitat and promote or impede the life of certain organisms (Ikawa et al., 1998).

Three-dimensional turbulence is thought to mostly have an homogenizing effect, smearing sharp gradients and promoting super-diffusive separation in time of initially close trajectories. The relative eddy diffusivity is expected to grow as the $4 / 3$ power of the separation distance $R(t)$, namely $D(R) \equiv$ $\mathrm{d}\left\langle R^{2}(t)\right\rangle / \mathrm{d} t \sim R^{4 / 3}$, and the separation distance hence grows as $\left\langle R^{2}(t)\right\rangle \simeq t^{3}$, as suggested by Richardson in his pioneering work (Richardson, 1926; Falkovich et al., 2001). Beyond the case of three-dimensional turbulence, the Richardson 4/3 law is observed also in the case of anisotropic relative dispersion, e.g. in the presence of a zonal mean shear and a meridional random walk (Bennett, 1987).

While the mathematical formulation of the problem of turbulent dispersion can be considered established (Bennett, 2005; Garrett, 2006), observations reported by experimental studies are much less clear (see e.g. LaCasce, 2010; Okubo, 1971; Morel and Larchevêque, 1974; Er-el and Peskin, 1981; Berti et al., 2011). This is only partly due to the inherent difficulties of performing float or dye concentration experiments in the ocean. Much of the uncertainty is due to the complex nature of the flow, and the relevance of non-ideal features associated with anisotropies and inhomogeneities, in addition to temporally or spatially local effects such as wind waves, and tidal and inertial fluctuations. 
From float trajectories analysis, Ollitrault and collaborators (2005) found that for pairs of particles, initially separated by a few kilometres, the relative diffusivity followed the $4 / 3$ law for separation distances between 40 and $300 \mathrm{~km}$. The experiment was conducted in the central part of the North Atlantic, where the Rossby internal radius of deformation is about $25 \mathrm{~km}$. Coastal region experiments are puzzling. Some, such as Ohlmann et al. (2012), tend to support the existence of an exponential regime, beside or instead of the power-law one, while others, such as Schroeder and et al. (2012), show results conflicting with the exponential behaviour.

More recently, Poje et al. (2014) performed a Lagrangian experiment in the Gulf of Mexico, the GLAD experiment, deploying an unprecedented number of CODE drifters. In particular, they quantified pair dispersion rates in agreement with the Richardson law. Also, they pointed out that the submesoscale dispersion rates when based on ocean model or altimetric velocities are largely underestimated with respect to the observed ones.

When dealing with ocean diffusion, there is a huge experimental gap between buoyant/surface/two-dimensional processes and three-dimensional ones, the former being much more observed than the latter. Lagrangian diffusion due to horizontal velocity variations across the three-dimensional structure of the mixing layer is clearly crucial to the transport and fate of sediments, biological material such as chlorophyll, and contaminants suspended in the ocean (Young et al., 1982; Steinbuck et al., 2011). In particular, the role of submesoscale and small-scale turbulent motions is at the core of recent research (see e.g. Lévy et al., 2012; Zhong and Bracco, 2013). Its full assessment is hampered by the lack of high-frequency, multiscale measurements of the velocity field within and below the mixing layer.

To simplify the problem, one might be tempted to use depth-averaged currents for predicting horizontal dispersion, so neglecting vertical shear effects. As is discussed in what follows, this approach can be misleading and can have some important practical drawbacks when estimating the dispersion of 3-D tracers.

The effect of vertical shear on the horizontal dispersion was first experimentally investigated by Okubo $(1968,1971)$. Later, LaCasce and Bower (2000) discussed it in relation to the dispersion of subsurface floats in the North Atlantic. On the basis of estimates inferred from the mean flow and not from the fluctuating velocities, it is argued that vertical shear is expected to be much less important than horizontal shear for the oceanic horizontal diffusion (LaCasce and Bower, 2000).

From the numerical modelling point of view, being able to simulate Lagrangian dispersion in the ocean has great relevance, but it is a delicate task because of the finite resolution of the circulation models, and more fundamentally because of the nonlinear character of the dynamics. Indeed, when dealing with basin-scale models, not only the mixing layer dynamics is often missing, but also the velocity field features from sub- to mesoscales are poorly resolved both temporally and spatially. In this regard, various techniques (Griffa, 1996; Berloff and McWilliams, 2002; Haza et al., 2007, 2012) have been developed to model the submesoscale or unresolved velocity components which, nonetheless, play an important role for tracer dispersion.

In this paper, we focus on the role of vertical shear as an important mechanism promoting the horizontal diffusion in the upper ocean. By vertical shear, we mean the vertical gradients of the horizontal velocities. The approach here considered consists in combining observation and model data to assess the effect of vertical shear for the tracer horizontal relative dispersion. Observation data come from Acoustic Doppler Current Profilers (ADCPs) deployed in a narrow region of the South Mediterranean. Numerical data come from the Mediterranean Forecasting System (MFS) model, and are supplemented with the use of deterministic kinematic models (Palatella et al., 2014; Lacorata et al., 2014), to parametrize poorly resolved mesoscale motions, or unresolved processes in General Circulation Models (GCMs).

The Kinematic Lagrangian Model (KLM) here adopted can be 2-D, to better account for the horizontal dispersion due to mescoscale eddies, or 3-D, to simulate vertical turbulent-like motions in the ocean mixing layer. Both dynamics are often underestimated in GCMs. Although our primary interest is in the former situation, we will discuss both.

The paper is organized as follows. In Sect. 2, we compare in situ observations of vertical gradients of the horizontal velocities with measures obtained from the MFS. The comparison highlights that velocity gradient correlation times derived from MFS are considerably larger than the observed ones: such anomalous temporal persistence of the vertical shear is responsible for an enhanced relative dispersion rate, which is possibly an artifact of the low temporal resolution of the model. Since the comparison is performed in one location only, its results might not be of general validity. Rather, they point to differences that can arise between model and data, which in turn motivate the sensitivity study presented in Sect. 3, where we discuss the relative dispersion properties of neutrally buoyant tracers by means of numerical simulations. We show that, by a suitable implementation of the kinematic model, the anomalous shear effect can be overcome. Section 4 contains the final remarks and perspectives.

\section{Vertical shear statistics: experimental versus numerical data}

We analyse the profiles of the horizontal velocities recorded with two ADCPs working at $300 \mathrm{kHz}$. These have been deployed on the continental shelf of the South Mediterranean: the first one is located at the following position: $31.91^{\circ} \mathrm{N}$, $30.58^{\circ} \mathrm{E}$, the second one at the close position $31.92^{\circ} \mathrm{N}$, $32.00^{\circ} \mathrm{E}$ (see Fig. 1). Both instruments are bottom-mounted at the depth of $104 \mathrm{~m}$; currents $(U(Z, t), V(Z, t))$ are uni- 

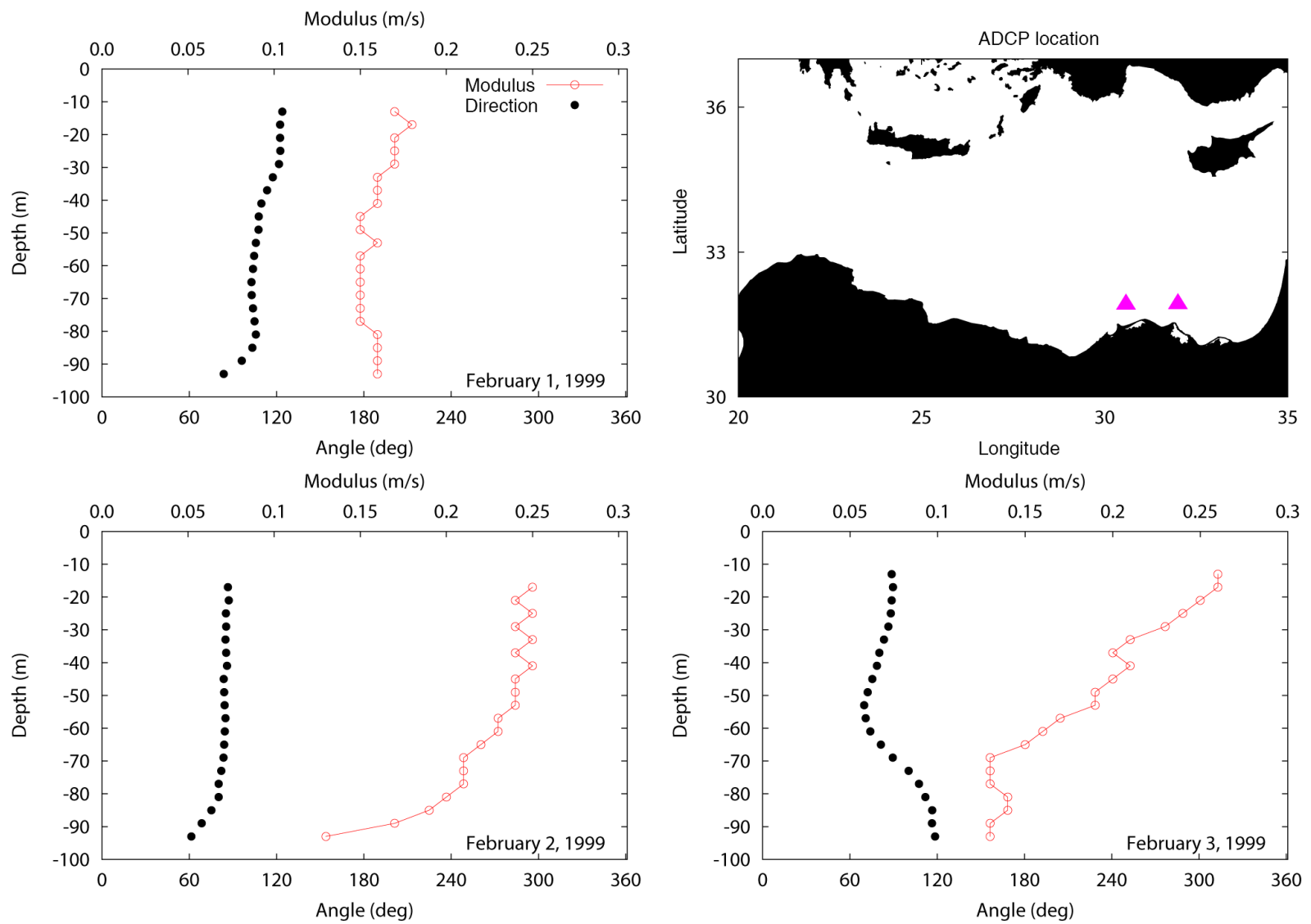

Figure 1. (Colour online.) Three instances of the observed instantaneous current profiles of the horizontal velocities, from the ADCP located at $31.91^{\circ} \mathrm{N}, 30.58^{\circ} \mathrm{E}$; empty red circles: velocity modulus; black filled circles: direction from the north. In the top right panel, the small purple triangles indicate the ADCP locations.

formly measured between $Z=-13 \mathrm{~m}$ and $Z=-93 \mathrm{~m}$, the spacing is $\delta Z=4 \mathrm{~m}$. The vertical component of the velocity is not directly available. The measurement database covers the period from 1 February 1999 until 11 February 2000: for each day we have on average 144 profiles (10 min interval). We analyse data separating them into two time intervals: I1 refers to February-April 1999; I2 refers to December 1999-February 2000. In both periods, the thermocline is about $80 \mathrm{~m}$ deep. Figure 1 shows three examples of the recorded profiles, together with the ADCP locations. ADCP results on vertical shear are significant with respect to measurement errors. We note however that the data set can be considered of good quality, both in terms of the statistical accuracy and of the measurement conditions: only seldom may low acoustic backscatter and diurnal migration of the scattering source cause noise in the data. By averaging over a large number of profiles, we can reduce ADCP velocity uncertainties.

In situ measurements are compared to current data, at the same locations and for the same period, extracted from MFS (Tonani et al., 2008). The MFS model uses the primitive equations with the Boussinesq, hydrostatic, and incompress- ible approximations written in spherical coordinates. Grid resolutions are of $1 / 16^{\circ} \times 1 / 16^{\circ}$ in the horizontal directions $(\approx 6.5 \mathrm{~km})$, with 72 vertical levels. The unevenly spaced levels have a thickness ranging from $3 \mathrm{~m}$ near the surface, to $300 \mathrm{~m}$ at the bottom. The first level is $1.5 \mathrm{~m}$ deep and the last is about $5000 \mathrm{~m}$ deep. If we estimate the first internal Rossby radius of deformation as of the order of $10 \mathrm{~km}$ on average, then MFS is an eddy-permitting model for the Mediterranean Sea. Current data outputs are daily.

Our primary interest being the vertical shear, we adopted the following procedure in the statistical analysis of ADCP current profiles:

- we remove the mean velocity component from the current measurements at different levels, obtaining $U^{\prime}(Z, t)$ and $V^{\prime}(Z, t)$;

- for each $\delta Z$, the time series of the vertical gradients of the horizontal components are constructed as $\gamma_{x}(Z, t)=$ $\left[U^{\prime}(Z, t)-U^{\prime}(Z-\delta Z, t)\right]$, and $\gamma_{y}(Z, t)=\left[V^{\prime}(Z, t)-\right.$ $\left.V^{\prime}(Z-\delta Z, t)\right]$ 

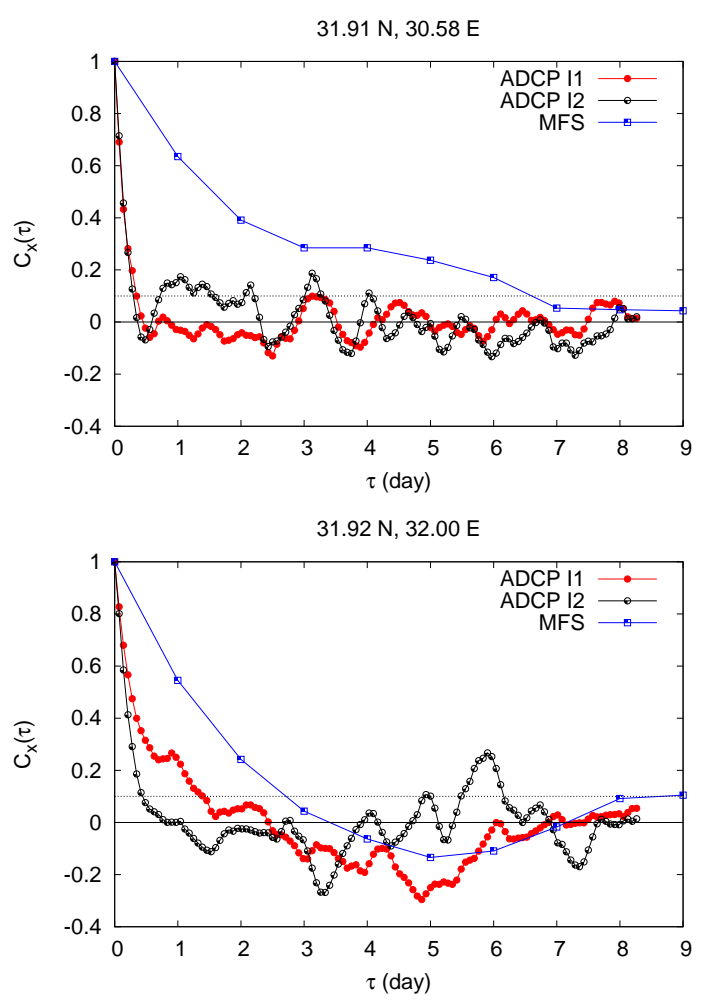

Figure 2. Log-lin plot of the velocity gradient autocorrelation functions versus the time lag. All data refer to the $\gamma_{x}^{\prime}(t)$ component. Top plot is for the ADCP located at $31.91^{\circ} \mathrm{N}, 30.58^{\circ} \mathrm{E}$; bottom plot is for the ADCP located at $31.92^{\circ} \mathrm{N}, 32.00^{\circ} \mathrm{E}$. Symbols: filled circles are for ADCP data of the period I1, February-April 1999; empty circles for ADCP data of the period I2, December 1999-February 2000; squares are for the MFS data averaged over period I1 and I2. Dotted lines indicate the value 0.1 .

- the velocity gradient residual times series, $\gamma_{x}^{\prime}(Z, t)$ and $\gamma_{y}^{\prime}(Z, t)$, are obtained by removing the mean gradient, estimated over the whole time series.

We first calculate the auto-correlation function $C_{x, y}(\tau)$ separately for each velocity gradient component as

$C_{x, y}(\tau) \equiv \frac{\left\langle\left[\gamma_{x, y}^{\prime}\left(t_{0}+\tau\right) \gamma_{x, y}^{\prime}\left(t_{0}\right)\right]\right\rangle}{\left\langle\left[\gamma_{x, y}^{\prime}\left(t_{0}\right)\right]^{2}\right\rangle}$

where the average is performed over different choices of the initial record $t_{0}$, and over a few depths between $Z=$ $-20 \mathrm{~m}$ and $Z=-50 \mathrm{~m}$, to gain statistical accuracy. Currents at lower and larger depths have not been considered.

In Fig. 2, we compare the auto-correlation functions obtained from the ADCPs with those of the MFS fields, for the same days and the same locations. Data exhibit specific behaviour depending on the location and on the averaging period. However, general features can also be found. The ADCP $C_{x, y}(\tau)$ curves are oscillatory, which makes the de- termination of the correlation time

$\mathcal{T}_{c}=\int_{0}^{\infty} C(\tau) \mathrm{d} \tau$

quite difficult. In the absence of a well-converged integral, a possible choice is to estimate the value of $\mathcal{T}_{c}$ from the time lag at which the curve attains the value 0.1 . Clearly such extrapolation is quite rough and an error of the order of $10 \%$ should be considered. ADCP data show that vertical shear components usually persist over a correlation time $\mathcal{T}_{c}^{\mathrm{ADCP}} \simeq 0.5$ day or less.

For MFS curves, the situation is rather different: in one case, the curve never really attains zero; in the other case, it does on a time lag $\mathcal{T}_{c}^{\mathrm{MFS}} \simeq 5$ days, so about 10 times bigger. This observation suggests that at least this GCM might overestimate the temporal persistence of velocity gradients, unrealistically increasing the effect of the shear on the horizontal dispersion.

Beside the characteristic timescales, it is useful to quantify the amplitude of velocity gradient fluctuations. Figure 3 shows the behaviour of the probability distribution functions (PDFs) of the vertical shear components; PDFs are normalized to have unit variance. The PDFs are extracted from the $\mathrm{ADCP}$ at $31.92^{\circ} \mathrm{N}, 32.00^{\circ} \mathrm{E}$, averaging over the periods I1 and I2; the same is repeated for MFS data interpolated at the same location. If we directly compare ADCP with MFS data, it appears that the former has a larger variance, which is clearly associated with the fact that MFS velocities do not have small-scale and high-frequency variability. Additionally, the ADCP PDF has fat tails, the fingerprint of a turbulent-like dynamics. Taking into account such variability could be important for the modelling of ocean mixing layer dynamics (Fox-Kemper and Ferrari, 2008).

However, if we compare daily averaged ADCP with MFS data, the cores of the unitary variance PDFs are very similar (not shown): this implies that at least for mean fluctuations, experimental ADCP and numerical MFS data account for dynamical behaviours having the same mean amplitudes.

\section{Lagrangian dispersion: the effect of vertical shear}

We start by considering a neutrally buoyant tracer particle whose position is given by the three-dimensional vector $\boldsymbol{X}(t)$. The trajectory is assumed to evolve according to the Lagrangian equation

$\frac{\mathrm{d} \boldsymbol{X}}{\mathrm{d} t}(t)=\boldsymbol{U}(\boldsymbol{X}, t)+\boldsymbol{u}(\boldsymbol{X}, t)$,

where the velocity field is simply decomposed in a largescale term, $\boldsymbol{U}(\boldsymbol{x}, t)$, and a small-scale contribution $\boldsymbol{u}(\boldsymbol{x}, t)$. We define the former, $(U, V, W)$, as the resolved component of the GCM, and the latter, $(u, v, w)$, as the unresolved or poorly resolved component. 


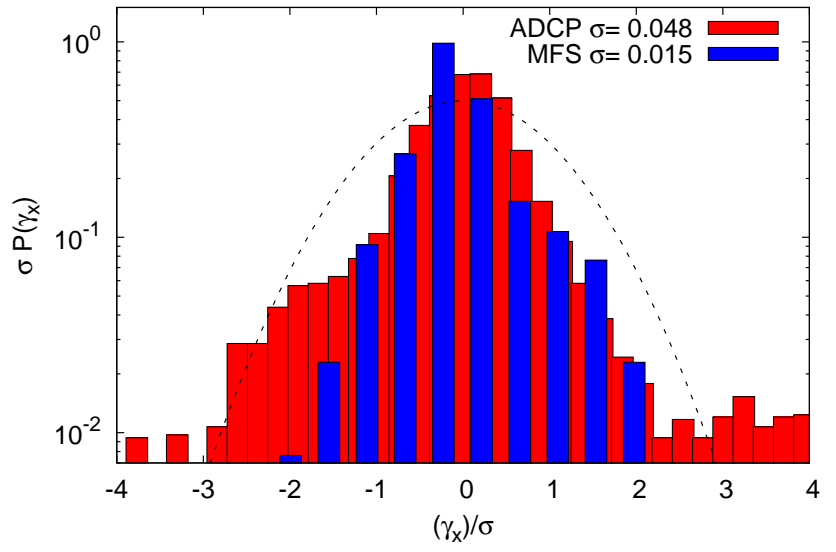

Figure 3. Lin-log plot of PDFs of the vertical shear component $\gamma_{x}^{\prime}$. PDFs are normalized to have unit variance. Red boxes are for the ADCP data at location $31.92^{\circ} \mathrm{N}, 32.00^{\circ} \mathrm{E}$; blue boxes are for the MFS data interpolated at the same location; the dashed curve is a normal distribution.

When considering Lagrangian dispersion, the problem is easily reformulated in terms of the time evolution of the pair separation vector $\boldsymbol{R}(t) \equiv \boldsymbol{X}_{i}(t)-\boldsymbol{X}_{j}(t)$, where the indices $i, j=1, \ldots, n$ indicate the tracer particles, and $i \neq j$ :

$$
\frac{\mathrm{d} \boldsymbol{R}}{\mathrm{d} t}(t)=\boldsymbol{\Delta}_{\boldsymbol{R}} \boldsymbol{U}(\boldsymbol{R}, t)+\boldsymbol{\delta}_{\boldsymbol{R}} \boldsymbol{u}(\boldsymbol{R}, t) .
$$

Two particles at mutual distance $R_{0}=|\boldsymbol{R}(t=0)|$ start to separate because of a non-zero velocity fluctuations at that scale. Depending on the value of $R_{0}$ and on the local dynamics, such velocity fluctuations can be ascribed to very different flow motions. Let us consider the simple situation of two particles, P1 and P2, located in the ocean mixing layer and initially separated along the vertical direction only, i.e. $\boldsymbol{R}_{0}=\left(\simeq 0, \simeq 0, R_{0}\right)$. In the absence of vertical shear, and taking into account that vertical velocities are very small, these particles will keep their initial separation almost unchanged so that $R(t) \simeq R_{0}$, or would separate very slowly. As a result horizontal diffusion will be very weak.

The situation is different when e.g. particles have the chance to experience for some time a mean vertical shear. If this is the case, with $U\left(Z_{1}, t\right) \neq U\left(Z_{2}, t\right)$ and/or $V\left(Z_{1}, t\right) \neq$ $V\left(Z_{2}, t\right)$, particles will start separating. This is better illustrated in Fig. 4, which shows that vertical shear causes horizontal pair dispersion. It is clear that the vertical shear is bounded by two opposite situations: on the one hand, a mean shear, i.e. a shear profile with very long correlation time as happens for example in the presence of strong background currents; on the other hand, a fluctuating vertical shear due to turbulent motions and hence rapidly changing in time. As we have seen in the previous section, the situation in the mixing layer of the Mediterranean sea is in between these two extrema, and the typical timescale turns out to be less than or of the order of 1 day. The MFS estimate is much longer, as a result of the low temporal resolution of the vertical gradients of the horizontal velocities in the model.

\subsection{Numerical simulations of Lagrangian dispersion}

We discuss different sets of numerical simulations based on the velocity configurations of the MFS model, also supplemented by the use of the kinematic model to describe poorly resolved motions. Kinematic models can be adapted to the different dispersion regimes, namely exponential separation, turbulent dispersion, and standard diffusion. Their implementation hence depends on the specific dynamics and specific range of scales that one wants to describe. Here, we compute transport properties by introducing statistical Lagrangian motions for the mixing layer motions (3-D KLM), and separately for the poorly mesoscale motions (2-D KLM). By doing so, we demonstrate that (i) small-scale motions (due to the 3-D KLM) enabling tracer pairs to explore the whole mixing layer do not modify the MFS horizontal dispersion properties, due to the anomalous persistence of vertical gradients of the horizontal velocities in the MFS model, which overrides the small-scale fluctuations; (ii) differently, the horizontal relative separation resulting from the introduction of the 2-D KLM is fast enough to become dominant with respect to the anomalous shear effect produced by the MFS solution.

Lagrangian numerical simulations are performed using as large-scale velocities the zonal $U(\boldsymbol{x}, t)=U_{\mathrm{MFS}}$ and meridional $V(\boldsymbol{x}, t)=V_{\mathrm{MFS}}$ components provided by MFS, and interpolated at particle positions; the velocity vertical component $W(\boldsymbol{x}, t)$ is not explicitly available in the MFS data file considered, and we did not consider it. To take into account unresolved fluctuations, we adopt a strategy in terms of a Lagrangian deterministic kinematic modelling.

In the following, we first describe the 3-D KLM which is meant to account for the transport and mixing in the upper layer of the ocean; then we introduce the 2-D KLM accounting for the poor-resolution of mesoscale horizontal motions. More details on the KLM definition and implementation can be found in Palatella et al. (2014) and Lacorata et al. (2014).

\section{The 3-D KLM}

In compact form, the three-dimensional velocity field of the $\mathrm{KLM},(u, v, w)$, is defined as the curl of the vector potential $\boldsymbol{\Phi}(\boldsymbol{x}, t)$ :

$$
\begin{aligned}
& u(\boldsymbol{X}, t)=\frac{\partial \Phi_{1}(\boldsymbol{x}, t)}{\partial z}, \\
& v(\boldsymbol{X}, t)=-\frac{\left.\partial \Phi_{2}(\boldsymbol{x}, t)\right)}{\partial z}, \\
& w(\boldsymbol{X}, t)=-\frac{\left.\partial \Phi_{1}(\boldsymbol{x}, t)\right)}{\partial x}+\frac{\partial \Phi_{2}(\boldsymbol{x}, t)}{\partial y},
\end{aligned}
$$

hence $\boldsymbol{u}(\boldsymbol{x}, t)$ is divergence-free by definition. The vector potential itself $\boldsymbol{\Phi}=\left(\Phi_{1}(x, z, t), \Phi_{2}(y, z, t), 0\right)$ has two compo- 


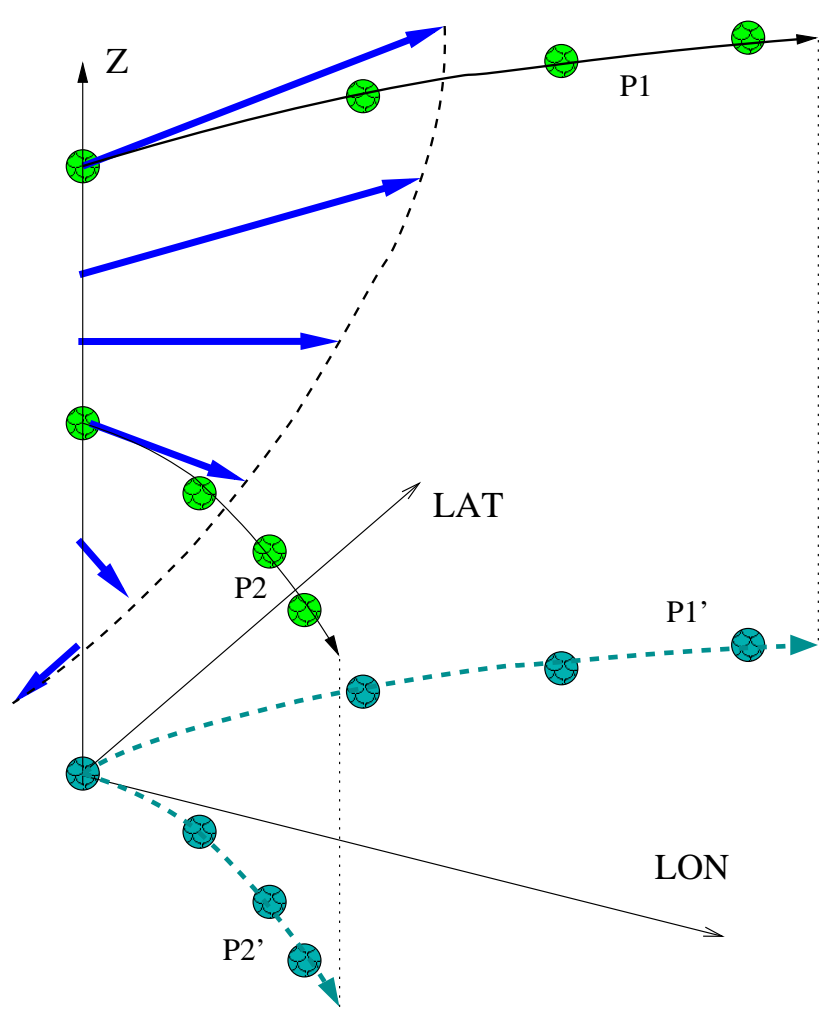

Figure 4. An illustration of the effect of vertical shear on the mean horizontal dispersion of two particles, P1 and P2, initially separated along the vertical direction.

nents and depends on the three spatial variables and the time variable, as follows:

$\Phi_{1}(x, z, t)=\frac{A}{\widehat{k}} \sin [k(x-s \sin (\omega t))] \sin [\widehat{k}(z-s \sin (\omega t))]$

$\Phi_{2}(y, z, t)=\frac{A}{\widehat{k}} \sin [k(y-s \sin (\omega t))] \sin [\widehat{k}(z-s \sin (\omega t))]$,

in analogy with chaotic cellular flows (Solomon and Gollub, 1988; Crisanti et al., 1991; Lacorata et al., 2008). Further, the suppression of the vertical dynamics below the mixing layer is included in terms of a damping term $\Upsilon(z)=\exp (-|z| / L)$, multiplying the vector potential $\boldsymbol{\Phi}$. Such exponential relaxation term guarantees that KLM velocities go to zero at depths much larger than the length scale $L$, where $L$ is of the order of the mixing layer depth.

The explicit form of the velocity components of the 3-D model then results as follows:

$u_{3-\mathrm{D}}=e^{-|z| / L} \times$

$[A \sin [k(x-s \sin (\omega t))] \cos [\widehat{k}(z-s \sin (\omega t))]$

$-\frac{A}{L \widehat{k}} \sin [k(x-s \sin (\omega t))] \sin [\widehat{k}(z-s \sin (\omega t))]$,

$v_{3-\mathrm{D}}=e^{-|z| / L} \times$

$[-A \sin [k(y-s \sin (\omega t))] \cos [\widehat{k}(z-s \sin (\omega t))]$
$\left.+\frac{A}{L \widehat{k}} \sin [k(y-s \sin (\omega t))] \sin [\widehat{k}(z-s \sin (\omega t))]\right]$,

$w_{3-\mathrm{D}}=e^{-|z| / L} \times$

$\left[-A_{\widehat{k}}^{k} \cos [k(x-s \sin (\omega t))] \sin [\widehat{k}(z-\sin (\omega t))]\right.$

$\left.+A_{\widehat{k}}^{k} \cos [k(y-s \sin (\omega t))] \sin [\widehat{k}(z-s \sin (\omega t))]\right]$

$+e^{-|z| / L} \times$

$\left[A \frac{k}{L \widehat{k}} \sin [k(x-s \sin (\omega t))] \sin [\widehat{k}(z-\sin (\omega t))]\right.$

$\left.-A \frac{k}{L \widehat{k}} \sin [k(y-s \sin (\omega t))] \sin [\widehat{k}(z-s \sin (\omega t))]\right]$

In the expressions above, $A$ is the velocity amplitude, $k=$ $2 \pi / l_{0}$ is the horizontal wavenumber associated with the wavelength $l_{0}$ of the flow, $\widehat{k}=2 k$ is the vertical wavenumber assumed to be twice the horizontal wavenumber for isotropy, $t_{c}=l_{0} / A$ is the convective timescale, and $s$ and $\omega$ are amplitude and pulsation of the time-dependent oscillating terms.

In order to simulate the mixing-layer dynamical effect of a multiscale velocity field with a turbulent-like behaviour, as is customary we superimpose $n$ different modes. For the 3-D KLM, we use $n=5$ and the velocity field of Eq. (7) becomes the sum of different terms with $A=A_{i} ; k=k_{i} ; \omega=\omega_{i} ; s=$ $s_{i}$ for $i=1, \ldots, 5$. The small spatial scales $l_{i}$ and their associated fast timescales $t_{i} \simeq l_{i} / A$ are chosen to reproduce, on average, the dynamical properties within the mixing layer. Pulsations of the perturbations, responsible for the Lagrangian chaotic behaviour, are dimensionally chosen, $\propto 1 / t_{i}$. In particular for the 3-D KLM, we use these values for the model parameters:

$\left\{\begin{array}{l}l_{n}=\{25.0,33,4,50.0,70.7,100\} \mathrm{m} \\ k_{n}=2 \pi / l_{n} ; A_{n}=\left(\epsilon l_{n}\right)^{1 / 3} \mathrm{~m} / \mathrm{s} \\ \omega_{n}=2 \pi A_{n} / l_{n} ; \\ L=100 \mathrm{~m}, \quad \varepsilon=10^{-5} \mathrm{~m}^{2} \mathrm{~s}^{-3} .\end{array}\right.$

Finally, $\varepsilon$ is the kinetic energy dissipation rate and it is used as the main parameter through the dimensional relation $|\boldsymbol{u}|^{3} \simeq L \varepsilon$, valid for the turbulent-like regime (Frisch, 1995).

\section{The 2-D KLM}

The KLM for the unresolved mesoscale motions is built up, in analogy with the 3-D model, in terms of an ensemble of horizontal cells forming a 2-D regular lattice (Palatella et al., 2014).

The explicit form of the 2-D sub-grid velocity is

$$
\begin{aligned}
& \left.u_{2-\mathrm{D}}=\sum_{j=1}^{6} A_{j} \sin \left[k_{j} x-k_{j} s_{j} \sin \left(\omega_{j} t\right)\right)\right] \\
& \cos \left[k_{j} y-k_{j} s_{j} \sin \left(\omega_{j} t+\theta\right)\right], \\
& \left.v_{2-\mathrm{D}}=-\sum_{j=1}^{6} A_{j} \cos \left[k_{j} x-k_{j} s_{j} \sin \left(\omega_{j} t\right)\right)\right]
\end{aligned}
$$




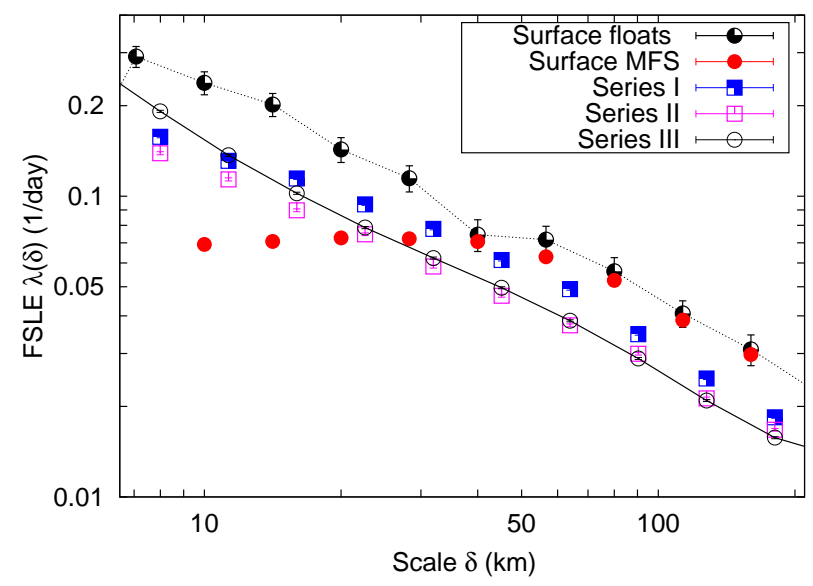

Figure 5. (Colour online.) Log-log plot of the finite-scale Lyapunov exponent $\lambda(\delta)$ versus the separation scale $\delta$; black filled circles: surface drifters; red filled circles: MFS surface particles (both after Lacorata et al., 2014); blue filled squares: Series I, that is the MFS model for particle pairs at fixed depths; purple empty squares: Series II, that is the MFS model plus the 3-D KLM; black empty circles with solid line: Series III, as Series II plus the 2-D KLM. Error bars, often smaller than the symbols themselves, are estimated from the standard deviation of the FSLE.

$\left.\sin \left[k_{j} y-k_{j} s_{S} \sin \left(\omega_{j} t+\theta\right)\right)\right]$,

where the subscript is meant to stress that the 2-D KLM is not equal to the 3-D KLM in the absence of the vertical velocity. The choice of parameters for the 2-D model is the following:

$\left\{\begin{array}{l}l_{j}=\{10.0,14.120 .0,28.0,40.0,56.5\} \mathrm{km} \\ s_{j}=l_{j} / 10, \theta=\pi / 4 \\ \varepsilon=10^{-9} \mathrm{~m}^{2} \mathrm{~s}^{-3}\end{array}\right.$

and $A_{j} \propto\left(\varepsilon l_{j}\right)^{1 / 3}, \omega_{j}=2 \pi A_{j} / l_{j}$, and $k_{j}=2 \pi / l_{j}$. As in Lacorata et al. (2014), these parameters have been tuned in order to numerically obtain a horizontal dispersion with the same statistical properties (more precisely the same finite-scale Lyapunov exponent, FSLE) as the actual surface drifters (see above the points labelled as "Surface floats" in Fig. 5). It is important to note the difference between the choice of parameters of the 2-D and 3-D models: they act on a well-separated range of scales, and mimic different effects, as mentioned above.

\section{Numerical experiment set-up}

We performed three series of numerical simulations releasing $N_{\text {pair }} \simeq 50000$ pairs of neutrally buoyant particles. In all series, pairs are initially homogeneously distributed in the whole Mediterranean Sea, $10 \mathrm{~km}$ offshore from the coast.

An elastic collision takes place when particles meet the domain boundaries. Within each pair, particles start at the same latitude and longitude position, but they are vertically separated: one particle starts at $z=-3 \mathrm{~m}$ below the surface, the other at $z=-43 \mathrm{~m}$, hence $\boldsymbol{R}_{0}=(0,0,40)$. Simulations are carried out for 1 year (from 1 January to 31 December 2009), and the integration time step is $\mathrm{d} t=120 \mathrm{~s}$.

The three series of simulations are characterized as follows:

- Series I: the KLM velocity is absent and particles keep their initial depth unchanged throughout the entire simulation. This is quite far from realistic conditions; however, this numerical experiment is useful to quantify the effect of the vertical shear solely due to the mesoscale MFS model dynamics.

- Series II: the 3-D KLM term is switched on, with the parameters shown in Eq. (8). As a result of the presence of the sub-grid-scale turbulent dynamics, particles can also move vertically (between the surface and a depth scale of the order of $L$ ).

- Series III: this differs from the Series II due to the fact that in addition to the 3-D KLM, the 2-D KLM model is also implemented: $\boldsymbol{u}=\boldsymbol{u}_{3-\mathrm{D}}+\boldsymbol{u}_{2-\mathrm{D}}$. The 2-D KLM is essential in simulating the mesoscale structures that are not completely resolved by the MFS model.

In addition, we also compare results from these runs with results obtained in Lacorata et al. (2014), considering MFS surface tracked particle and drifter trajectories. Drifter data belong the Mediterranean SeaIn-Situ Near Real Time Observations (database INSITU_MED_NRT_OBSERVATIONS_013_035 available on http://marine.copernicus.eu/). These are surface buoys, drogued at a nominal depth of $15 \mathrm{~m}$ (Poulain et al., 2012). The comparison with surface drifters is here used as a benchmark for the 2-D kinematic model.

\subsection{Lagrangian dispersion diagnostic: the FSLE}

The most natural way to quantify Lagrangian dispersion statistics is in terms of the moments $\left\langle\boldsymbol{R}^{p}(t)\right\rangle$ of the pair separation PDF $P(\boldsymbol{R}, t)$ (LaCasce, 2010; Biferale et al., 2014), measuring the probability to observe a pair separated by the distance vector $\boldsymbol{R}$ at time $t$. Standard observables are the moment of order 2, the mean square particle separation $\left\langle\boldsymbol{R}^{2}(t)\right\rangle$, and its time derivative, i.e. the relative diffusivity $D(\boldsymbol{R}, t)$. Alternatively one can use the FSLE (Boffetta et al., 2000). The advantage of this choice, often exploited in ocean dispersion applications (LaCasce, 2008), is that different dispersion regimes are disentangled and crossover effects are minimized. Furthermore, the finite-time Lyapunov exponent (FTLE) is also used to detect Lagrangian coherent structures in ocean dynamics applications (Haller, 2000; Sulman et al., 2013). A discussion of the use of scale-dependent indicators in Lagrangian dispersion problems can be found in Berti et al. (2011), while a direct comparison of FSLE and FTLE for the identification of transport barriers can be found in Boffetta et al. (2001). 
The measure of FSLE consists of fixing a set of threshold scales, $\delta_{n}=\rho^{n} \delta_{0}$, where $\rho>1, n=1,2,3, \ldots$ and $\delta_{0}$ can be chosen of the order of the initial pair separation. We then need to calculate the time, $T(\delta)$, it takes for the pair separation distance $R(t)$ to change from $\delta_{n}$ to $\delta_{n+1}$. By averaging over the particle pair ensemble, we obtain the mean exit time, $\left\langle T_{\rho}\left(\delta_{n}\right)\right\rangle$, or mean doubling time if $\rho=2$. Formally we are calculating the first passage time. The FSLE has the dimension of an inverse of time and is defined as

$\lambda(\delta) \equiv \frac{1}{\langle T(\delta)\rangle} \ln \rho$.

If $\delta \rightarrow 0$, the FSLE no longer depends on the scale and coincides with the maximum Lyapunov exponent on the flow: this happens when particles separate exponentially in time. For finite separations, if relative dispersion is governed by a $\left\langle R^{2}\right\rangle \simeq t^{v}$ regime, then by dimensional analysis the FSLE is expected to scale as $\lambda(\delta) \simeq \delta^{-2 / \nu}$. Most relevant regimes are the case of standard diffusion, for which we expect $\lambda(\delta) \simeq$ $\delta^{-2}$; Richardson's diffusion, $\lambda(\delta) \simeq \delta^{-2 / 3}$; and ballistic or shear dispersion, with $\lambda(\delta) \simeq \delta^{-1}$.

Here, since we want to compare how the horizontal diffusion is influenced by the different flow realizations, in the FSLE we consider horizontal separations only.

In Fig. 5, we compare FSLE results from the three series and results from Lacorata et al. (2014) at the surface. First, we observe that surface drifter and MFS surface tracer data show a striking difference: while at large scale they have the same behaviour, at a scale $\delta \simeq 40 \mathrm{~km}$ they depart. In particular, for Lagrangian particles moving in the MFS velocity field, numerical simulations unrealistically suggest that it would take approximately the same time to reach a separation scale of the order of a few kilometres and a separation scale 10 times bigger. As has been previously observed, this discrepancy is due to both the coarse spatial resolution and the time averaging of any mesoscale model - see e.g. Haza et al. (2012) and references therein.

Note that the scale at which MFS surface tracers deviate from drifters is larger that the model resolution: this suggests that scale resolution is crucial for Lagrangian statistics.

How does vertical shear affect these results? Can the vertical shear substantially modify horizontal dispersion? We address these questions using numerical data from Series I, II, and III.

In Series I, the effect of the vertical shear onto the horizontal dispersion comes from the MFS model only. The associated FSLE curves clearly indicate that vertical shear is able to promote horizontal dispersion. Neutrally buoyant tracers moving at different depths experience velocity differences: as a result they start to separate already at very small scales.

In Series II, the 3-D KLM terms are switched on, and particles vertically explore the whole mixing layer. The obtained FSLE curve is very similar to that of Series I, and in particular it turns out to be slightly below the latter. This finding is somehow surprising since, thanks to the introduction of small-scale turbulent-like motions, tracer pairs can explore the whole mixing layer. However, the fluctuations of the 3-D KLM do not substantially modify the horizontal pair dispersion, and actually they make it slightly slower in the present case. This suggests that the dominant effect is the one associated with the MFS vertical shear.

In Series III, both the 3-D and the 2-D KLM are switched on. The resulting FSLE is larger than that of Series II at any scale. This means that the most important dynamical correction to the MFS model is that associated with the 2-D KLM. Indeed, the dispersion effect induced by the mesoscale eddies inserted in the 2-D KLM overrides any other horizontal dispersion effects, including the one associated with the anomalous persistence of vertical gradients of the horizontal velocities in the MFS model.

We can summarize the results of the numerical simulations as follows. By comparing the horizontal dispersion of the bare MFS model with Mediterranean surface data, one sees that actual drifter pair dispersion follows a turbulentlike behaviour, whereas modelled surface trajectories separate more slowly and at a nearly constant rate. A way to solve this mismatch is to add a 3-D kinematic model, enabling vertical shear mixing and promoting surface horizontal dispersion also. However, the adoption of the 3-D KLM only does not seem an appropriate choice, since vertical gradients of the horizontal velocities have an anomalous temporal persistence, resulting in a spurious shear dispersion. Indeed, such persistence does not seem to have a counterpart in observational data, and we interpret this as an artifact of the poor temporal resolution of MFS model.

On the other hand, adding a two -dimensional kinematic model, one finds that the anomalous shear dispersion effects become practically negligible, being hidden by the more energetic dispersion processes occurring at the mesoscale. Clearly, mesoscale eddies are not pure 2-D structures, but they have a certain vertical development in the mixing layer. This implies that mesoscale turbulent dispersion is not a property of the surface layer only, but belongs to a whole vertical range of ocean layers. By adding the 2-D KLM for mesoscale eddies, one realizes that the effect is to have an efficient dispersion that covers the one due to mean vertical shear.

Finally, it is worth recalling that, as shown in Lacorata et al. (2014), the FSLE measured for the Mediterranean surface drifters previously discussed follows the Richardson diffusion behaviour $\lambda(r) \propto r^{-2 / 3}$ for $r \in[10: 100] \mathrm{km}$. This is consistent with the observed dispersion rates in the GLAD experiment, which spans however a much wider range of scales (Poje, 2014).

\section{Conclusions}

In this paper, we have discussed the effect of vertical shear onto the horizontal pair dispersion of tracer particles in a 
Mediterranean Sea model. Numerical simulations with the MFS model show that, differently from drifters, pairs released at the same depth tend to exponentially separate with a dispersion rate nearly constant over a wide range of scales, up to the mesoscale. At larger scales $(>100 \mathrm{~km})$, as soon as spatial correlation of the MFS velocity decay, the relative dispersion tends to a diffusive regime. However, if two trajectories having the same initial position are shifted in the vertical direction, then the horizontal dispersion rate grows as the separation tends to zero: this is the effect of the persistence of the vertical gradient of the horizontal velocities. This observation implies that, at spatial scales smaller or comparable with the mixing layer size, shear dispersion can be quite important. Its relevance might be under- or overestimated in the model, depending on whether vertical gradients of the current field change too fast or are anomalously persistent in time, respectively.

Now, the question arises, whether the proper small-scale ocean model velocity field is able to simulate the horizontal dispersion of a tracer having a 3-D structure in the mixing layer and below. The solution to this problem is very difficult, mainly because experimental data of 3-D tracer dispersion are not easily available. Different modelling solutions can be adopted to account for different problems, depending on whether the mesoscale, submesoscale or small scale is the relevant range of scales in the dispersion problem, none of which is straightforward.

For the specific problem of the effect of vertical shear, a different possibility, yet to be explored, is to build up an ad hoc Lagrangian small-scale kinematic model accounting for the locally homogeneous shear-dominated dynamics. In the context of large-eddy simulations, this has been done with the shear-improved sub-grid-scale models in the Eulerian framework (Lévêque et al., 2007). Future ocean experiments focusing on the dispersion properties of tracers having vertical structures, such as the chlorophyll field, are needed to reveal more about the dynamics and statistics at those spatial scales where shear might dominate.

Acknowledgements. We gratefully acknowledge useful discussions with G. Lacorata, who collaborated with us in the first part of the study. This work has been supported by SSD PESCA and RITMARE Research Projects (MIUR, the Italian Research Ministry). This study has been conducted using Marine Copernicus Products, that we acknowledge. The MFS current data are retrieved from MyOcean as MEDSEA_REANALYSIS_PHYS_006_004 myov04-med-ingv-cur-rean-dm. ADCP data were kindly delivered by the Italian Ente Nazionale Idrocarburi (ENI SpA). These data are collected as part of an industry-sponsored initiative and are currently proprietary. Technical support by Ing. F. Grasso at ISAC Lecce is kindly acknowledged.

Edited by: L. Kantha

\section{References}

Bennett, A. F.: A Lagrangian analysis of turbulent diffusion, Rev. Geophys., 25, 799-822, 1987.

Bennett, A. F.: Lagrangian Fluid Dynamics, Cambridge University Press, Cambridge, UK, 2005.

Berloff, P. and McWilliams, J.: Material transport in Oceanic gyres. Part 2: Hierarchy of stochastic models, J. Phys. Ocean., 32, 797830, 2002.

Berti, S., Alves Dos Santos, F., Lacorata, G., and Vulpiani, A.: Lagrangian Drifter Dispersion in the Southwestern Atlantic Ocean, J. Phys. Ocean., 41, 1659-1672, 2011.

Biferale, L., Lanotte, A. S., Scatamacchia, R., and Toschi, F.: Intermittency in the relative separations of tracers and of heavy particles in turbulent flows, J. Fluid Mech., 757, 550-572, 2014.

Boffetta, G., Celani, A., Cencini, M., Lacorata, G., and Vulpiani, A.: Non-asymptotic properties of transport and mixing, Chaos, 10, 50-60, 2000.

Boffetta, G., Lacorata, G., Redaelli, G., and Vulpiani, A.: Detecting barriers to transport: a review of different techniques, Phys. D, 159, 58-70, 2001.

Crisanti, A., Falcioni, M., Paladin, G., and Vulpiani, A.: Lagrangian chaos: Transport, mixing and diffusion in fluids, Il Nuovo $\mathrm{Ci}^{-}$ mento, 14, 1-80, 1991.

Davis, R.: Oceanic property transport, Lagrangian particle statistics, and their prediction, J. Mar. Res., 41, 163-194, 1983.

Durham, W. M., Climent, E., Barry, M., De Lillo, F., Boffetta, G., Cencini, M., and Stocker, R.: Turbulence drives microscale patches of motile phytoplankton, Nat. Commun., 4, 2148, doi:10.1038/ncomms3148, 2013.

Er-el, J. and Peskin, R.: Relative diffusion of constant-level ballons in the southern hemisphere, J. Atmos. Sci., 38, 2264-2274, 1981.

Falkovich, G., Gawȩdzki, K., and Vergassola, M.: Particles and fields in fluid turbulence, Rev. Mod. Phys., 73, 913-975, 2001.

Fox-Kemper, B. and Ferrari, R.: Parameterization of Mixed Layer Eddies. Part II: Prognosis and Impact, J. Phys. Oceanogr., 38, 1166-1179, 2008.

Frisch, U.: Turbulence, the legacy of A. N. Kolmogorov, Cambridge Univ. Press, Cambridge, UK, 1995.

Garrett, C.: Turbulent Dispersion in the Ocean, Prog. Ocean., 70, 113-125, 2006.

Griffa, A.: Applications of stochastic particle models to oceanographic problems, in: Stochastic Modelling in Physical Oceanography, edited by: Adler, R., Müller, P., and Rozovskii, B., 113128, Birkhauser, Boston, 1996.

Haller, G.: Finding finite-time invariant manifolds in twodimensional velocity fields, Chaos, 10, 99-108, 2000.

Haza, A. C., Piterbarg, L., Martin, P., Ozgokmen, T. M., and Griffa, A.: A Lagrangian subgridscale model for particle transport improvement and application in the Adriatic Sea using the Navy Coastal Ocean Model, Ocean Mod., 7, 68-91, 2007.

Haza, A. C., Ozgokmen, T. M., Griffa, A., Garraffo, Z. D., and Piterbarg, L.: Parameterization of particle transport at submesoscales in the Gulf Stream region using Lagrangian subgridscale models, Ocean Model., 42, 31-49, 2012.

Ikawa, T., Okubo, A., Okabe, H., and Cheng, L.: Oceanic diffusion and the pelagic insects Halobates spp. (Gerridae: Hemiptera), Mar. Biol., 131, 195-201, 1998.

Kantha, L. and Clayson, C.: Small Scale Processes in Geophysical Fluid Flows, Academic Press, 2000. 
LaCasce, J. H.: Statistics from Lagrangian observations, Prog. Ocean., 77, 1-29, 2008.

LaCasce, J. H.: Relative displacement probability distribution functions from balloons and drifters, J. Mar. Res., 68, 433-457, 2010.

LaCasce, J. H. and Bower, A.: Relative dispersion in the subsurface northatlantic, J. Mar. Res., 58, 863-894, 2000.

Lacorata, G., Mazzino, A., and Rizza, U.: 3D Chaotic Model for Subgrid Turbulent Dispersion in Large Eddy Simulations, J. Atmos. Sci., 65, 2389-2401, 2008.

Lacorata, G., Palatella, L., and Santoleri, R.: Lagrangian predictability characteristics of an Ocean Model, J. Geophys. Res.Oceans, 119, 8029-8038, 2014.

Lévêque, E., Toschi, F., Shao, L., and Bertoglio, J.-P.: Shearimproved Smagorinsky model for large-eddy simulation of wallbounded turbulent flows, J. Fluid Mech., 570, 491-502, 2007.

Lévy, M., Ferrari, R., Francks, P. J. S., Martin, A. P., and Rivìere, P.: Bringing physics to life at the submesoscale, Geophys. Res. Lett., 39, L14602, doi:10.1029/2012GL052756, 2012.

Morel, P. and Larchevêque, M.: Relative dispersion of constant level baloons in the $200 \mathrm{mb}$ general circulation, J. Atmos. Sci., 31, 2189-2196, 1974.

Ohlmann, J. C., LaCasce, J., Washburn, L., Mariano, A. J., and Emery, B.: Relative dispersion observations and trajectory modeling in the Santa Barbara Channel, J. Geophys. Res., 117, C05040, doi:10.1029/2011JC007810, 2012.

Okubo, A.: Some remarks on the Importance of the "Shear Effect" on Horizontal Diffusion, J. Ocean. Soc. Jap., 24, 60-69, 1968.

Okubo, A.: Oceanic diffusion diagrams, Deep-Sea Res., 18, 789802,1971

Ollitrault, M., Gabillet, C., and De Verdière, C.: Open ocean regimes of relative dispersion, J. Fluid Mech., 533, 381-407, 2005.
Palatella, L., Bignami, F., Falcini, F., Lacorata, G., Lanotte, A. S., and Santoleri, R.: Lagrangian simulations and interannual variability of anchovy egg and larva dispersal in the Sicily Channel, J. Geophys. Res.-Oceans, 119, 1306-1323, 2014.

Poje, A. E. A.: Submesoscale dispersion in the vicinity of the Deepwater Horizon spill, P. Natl. Aacad. Sci. USA, 111, 1269312698, 2014.

Poulain, P.-M., Menna, M., and Mauri, E.: Surface geostrophic circulation of the Mediterranean Sea derived from drifter and satellite altimeter data, J. Phys. Oceanogr., 42, 973-990, 2012.

Richardson, L. F.: Atmospheric diffusion on a distance-neighbour graph, Proc. R. Soc. Lond. A, 110, 709-737, 1926.

Schroeder, K., Chiggiato, J., Haza, A. C., Griffa, A., Ozgokmen, T. M., Zanasca, P., Molcard, A., Borghini, M., Poulain, P. M., Gerin, R., Zambianchi, E., Falco, P., and Trees, C.: Targeted Lagrangian sampling of submesoscale dispersion at a coastal frontal zone, Geophys. Res. Lett., 39, L11608, doi:10.1029/2012GL051879, 2012.

Solomon, T. H. and Gollub, J. P.: Chaotic particle transport in timedependent Rayleigh-Bénard convection, Phys. Rev. A, 38, 62806286, 1988.

Steinbuck, J. V., Koseff, J. R., Genin, A., Stacey, M. T., and Monismith, S. G.: Horizontal dispersion of ocean tracers in internal wave shear, J. Geophys. Res., 116, 1-16, 2011.

Sulman, M. H. M., Huntley, H., Lipphardt, B. J., and Kirwan, A.: Leaving Flatland: Diagnostics for Lagrangian coherent structures in three-dimensional flows, Phys. D, 258, 77-92, 2013.

Tonani, M., Pinardi, N., Dobricic, S., Pujol, I., and Fratianni, C.: A high-resolution free-surface model of the Mediterranean Sea, Ocean Sci., 4, 1-14, doi:10.5194/os-4-1-2008, 2008.

Young, W. R., Rhines, P., and Garrett, C. J. R.: Shear-flow dispersion, Internal Waves and Horizontal Mixing in the Ocean, J. Phys. Ocean., 12, 515-527, 1982.

Zhong, Y. and Bracco, A.: Submesoscale impacts on horizontal and vertical transport in the Gulf of Mexico, J. Geophys. Res.Oceans, 118, 5651-5668, 2013. 